\title{
Análisis de la producción científica de los artículos de la Revista Zootecnia Tropical del Instituto Nacional de Investigaciones Agrícolas (2006-2013)
}

\author{
Leomar José Montilla Peña \\ Universidad Politécnica Territorial de Lara Andrés Eloy Blanco - UPTAEB, Venezuela, República \\ Bolivariana de
}

ART I C L E

\begin{abstract}
Resumen
Objetivo: Conocer la producción científica agropecuaria y temas a fines publicados en la Revista Zootecnia Tropical del Instituto Nacional de Investigaciones Agrícolas, Venezuela. Método: Se realizó un análisis bibliométrico que incluye un estudio descriptivo y un análisis estadístico longitudinal desde el año 2006 hasta el 2013, se estudiaron 11 indicadores bibliométricos que van de acuerdo a la actividad científica y a los contenidos de los estudios. Resultados: Se analizaron un total de 380 publicaciones, el año de mayor productividad fue el 2008 con un total de 88 artículos científicos y 1 nota técnica, la media del índice de firmas/documentos es 4,43 durante todo el periodo estudiado, el 59,17\% de los documentos publicados provienen de instituciones relacionadas con la investigación agrícola, las temáticas registradas en los documentos publicados predominó una mayor centralidad y densidad en dos de los temas motores del campo científico. Conclusiones: El periodo estudiado muestra que la productividad de tipo documental está próxima a la misma y que se observar una mayor centralidad y densidad en los temas motores del campo científico, la tendencia se centra en la publicación de artículos científicos frente a otro tipo de trabajos, predominaron los temas de Fisiología y Bioquímica de la Planta, la productividad autoral mostro además que entre los autores más productivos el ranking oscila entre 26 a 8 contribuciones científicas.
\end{abstract}

Palabras clave

Bibliometria ; Producción científica ; Revista Zootecnia Tropica ; Venezuela

\section{Analysis of the scientific production of articles of Journal Tropical Zootecnia of the National Institute for Agricultural Research (2006-2013)}

\begin{abstract}
Objective: Evaluate agricultural production and scientific related topics published in the Journal Zootecnia Tropical the of the National Agricultural Research Institute, Venezuela. Method: Was performed a bibliometric analysis that includes a descriptive study and a longitudinal statistical analysis from 2006 to 2013 was performed 11 bibliometric indicators that go according to scientific activity and content of the studies were studied. Results: A total of 380 publications were analyzed, the most productive year was 2008 with a total of 88 scientific articles and one technical note, the average index of signatures / documents is 4.43 throughout the study period, the $59.17 \%$ of published documents come from institutions related to agricultural research, thematic registered in published documents predominated greater centrality and density in two of the engines topics of the scientific field. Conclusions: The period studied shows that productivity documentary is next to it and that greater centrality and density engine issues the scientific field is seen, the trend focuses on the publication of scientific papers over other types of work, predominated topics of Physiology and Biochemistry Plant, authorial productivity also showed that among the most productive authors ranking ranges from 26-8 scientific contributions.
\end{abstract}

Keywords

Bibliometrics ; Scientific production ; Journal Tropical Zootecnia ; Venezuela 


\section{Introducción}

El Instituto Nacional de Investigaciones Agrícolas (INIA) fue creado en el año 2000 y pasó a sustituir al Fondo Nacional de Investigaciones Agrícolas (FONAIAP), fundado en 1961. Al momento de realizarse la investigación el INIA contaba con 15 años transcurridos desde su instauración, siendo un periodo muy corto para una institución de este tipo. No obstante, ha logrado posicionarse entre las instituciones significativas en la investigación agrícola venezolana. Prueba de ello es por seguir apoyando los resultados de las investigaciones agrícolas a través de sus publicaciones científicas, preferentemente la Revista Zootecnia Tropical (ZT) que se publica desde el año 1983 y se encuentra indizada en bases de datos nacionales e internacionales como AGRIS, Bioline, Latindex, National Agricultural Library, Scielo, SIAN, entre otros.

En Venezuela se han desarrollado varias investigaciones para analizar bibliométricamente la Revista Zootecnia Tropical, esto se debe substancialmente porque quienes producen y utilizan la información científica escrita requieren que se realicen estudios bibliométricos para analizar la información registrada en los documentos. Basada en el uso de indicadores bibliométricos como método objetivo para valorar el comportamiento de la producción científica de las investigaciones realizadas por los autores y la colaboración que se genera entre ellos y a su vez servir como un método objetivo que complemente el proceso de arbitraje y revisión editorial (Spinak, 1996).

Por lo tanto, para lograr medir y evaluar objetivamente las actividades propias de la ciencia es necesario que se apliquen indicadores bibliométricos que permitan conocer la dinámica con que se desarrolla la ciencia a partir de los números de trabajos científicos publicados, la colaboración entre los científicos y las instituciones, determinar el impacto y visibilidad de las publicaciones dentro de determinada comunidad científica, entre otros (Pérez, 2014).

Sin embargo, por lo multidimensional de la ciencia, la misma debe ser evaluada a partir de indicadores multidimensionales que pongan en relieve las diferentes facetas de la actividad científica (Sancho, 1990). Tomando en cuenta los aspectos elementales, directos, inmediatos, complejos, concretos y reales de la objetividad para dilucidar las características de la producción científica de forma completa o sus particularidades aisladas.

También se requieren aplicar programas informáticos de mayor complejidad con el propósito de brindar una visualización de la información de los espacios documentales y los dominios de conocimientos que se generan, permitiendo así la toma decisiones correctas en el mejoramiento del flujo de información en las estructuras sociales (Montilla, 2012; Rubio, 1999; Torres, 2010; Chen, 2003; Sancho, Ob. Cit.).

Entre los análisis bibliométricos realizados a ZT realizados en Venezuela, puede citarse en primer lugar el realizado por Pérez (2003) que se ocupó del análisis temático y el consumo de información comprendido desde 1983 hasta 2002, donde caracteriza la producción anual de trabajos, análisis de la clasificación temática, vida media de las referencias de los artículos e índice de autocitas.

Seguidamente, Arenas y Romero (2003) ejercen un análisis bibliométrico en el que caracterizan el comportamiento de la producción científica desde 1985 hasta el 2001, estudiando los indicadores de oferta, impacto y visibilidad.

Luego, Chaparro y Marzal (2009) emplean un análisis bibliométrico desde 1985 hasta 2005 en donde analizan la producción científica, densidad bibliográfica, afiliación institucional, documentos citados, tipología de documentos citados, autocitas y colaboración científica.

Como hemos visto hasta ahora, los análisis bibliométricos empleados a ZT han servido para evaluar la calidad de los trabajos y la productividad científica originando un conjunto de resultados de gran utilidad y de aplicación práctica. No obstante, el análisis bibliométrico de la producción científica de ZT con indicadores multidimensionales no se ha realizado y que sean representados con técnicas alternativas que proporcionen una mejor visualización de la información.

Por otra parte, se realizó una búsqueda para definir las revistas que tratan sobre este tema en Venezuela, encontrando que esta es la única revista puntual que abarca el área, las otras están más centradas en los temas de prevención, diagnóstico y cura de las enfermedades de los animales. No se caracterizo esta área a nivel latinoamericano y caribeño ya que no es fácil definir unívocamente las revistas que tratan sobre el tema de la producción animal.

Considerando lo anteriormente planteado, el objetivo central de esta investigación fue evaluar bibliométricamente la producción científica publicada en los artículos de la revista ZT pertenecientes al período 2006 hasta el 2013, basado en once indicadores bibliométricos, utilizando el análisis descriptivo para brindar elementos informativos que permitan tomar acciones tanto a editores, investigadores y lectores. 


\section{Materiales y métodos}

El análisis bibliométrico se desarrolló en los artículos publicados en la Revista ZT del INIA Venezuela, se contó con los números de la revista en formato físico y digital, los artículos a texto completo en formato digital se obtuvo mediante el acceso al portal Web:

http://sian.inia.gob.ve/repositorio/revistas_ci/ZootecniaTropical/linea.htm

Se trata de un estudio descriptivo y de muestreo estadístico longitudinal comprendido desde el año 2006 hasta el año 2013. La variable estudiada fue la producción científica en la revista ZT. Se seleccionaron un total de 380 documentos publicados entre los números 24 al 31, se escogieron artículos científicos, notas técnicas y memorias en extensos que aportan el estado del arte y permiten conceptualizar la importancia de las publicaciones científica del área producción animal. Los datos obtenidos fueron tomados durante los meses de agosto-diciembre de 2015. Es importante destacar que el estudio bibliométrico de estos números de la Revista ZT, reflejaron resultados ampliados en función de los alcanzados al momento inicial de la investigación, debido a la no existencia de algunos números de la revista correspondiente con el año 2014 y muchos menos 2015, esto quizás se deba al no cumplimiento de sus publicaciones en los periodos correspondientes. Se seleccionaron 11 indicadores bibliométricos que representan el objetivo de esta investigación: producción documental, productividad por países, contribución por países, productividad institucional, productividad y distribución de documentos publicados por el INIA, productividad autoral, colaboración autoral, colaboración en las publicaciones, capacidad idiomática, categorías temáticas y categorías metodológicas.

Para el procesamiento de la información se llevo a cabo un laborioso trabajo de forma manual, una vez recopilado los datos, se procesaron al gestor de datos bibliográficos Endnote X2 para la posterior normalización de los datos, obteniendo una base de datos integrada de acuerdo a las preguntas formuladas en la investigación y los indicadores seleccionados permitiendo un recuento múltiple de acuerdo al campo seleccionado. La información fue recolectada y analizada desde el enfoque "análisis del dominio" (Hjørland y Albrechtsen, 1995) y el "análisis de co-palabras" (Callon, Law \& Rip, 1986) a través de la lectura de los resúmenes y del texto completo y con el análisis de coocurrencias las palabras clave para evitar posibles errores, lo que posibilito realizar un análisis cuantitativo y cualitativo de la producción científica de los artículos publicados durante este periodo.

Se determinó la distribución geográfica, al igual que la filiación institucional, teniendo en cuenta la procedencia geográfica y residencial del primer firmante del trabajo publicado, utilizando la técnica bibliométrica del recuento permitió la normalización de las instituciones debido a que no están debidamente normalizadas, lo que exigió indudablemente revisar uno a uno cada trabajo para evitar sesgos en la presente investigación.

La productividad autoral y la colaboración autoral se procesó a través del índice de autoría en la que se consideraron los firmantes principales y secundarios. Los autores también se agruparon teniendo en cuenta los grupos planteados por la Lotka (1926): pequeños productores (autores que producen un solo artículo), medianos productores (autores que producen entre dos y nueve artículos) y grandes productores (autores que producen entre 10 a más artículos). Posteriormente se elaboró un micro resumen curricular de los autores concentrados en el grupo de grandes productores tomando en cuenta los aspectos más importantes en su trayectoria profesional e investigativa.

El mismo procedimiento bibliométrico del recuento se siguió para la capacidad idiomática en cada uno de los documentos publicados, considerando el idioma en que está escrito el texto completo, el origen geográfico de los firmantes, el origen de residencia y sus capacidades idiomáticas para escribir en otros idiomas.

Para clasificar los contenidos en los 380 documentos seleccionados se trabajó con el análisis del dominio (Hjørland y Albrechtsen, Ob. Cit.), el análisis de co-palabras (Callon, Law \& Rip, Ob. Cit.) y se recurrió a la clasificación propuesta por del Sistema Internacional de Información sobre Ciencias y Tecnologías Agrícolas (AGRIS) (FAO, 1990), el código de objetos AGRIS y el tesauro AGROVOC (FAO, 1997) para la normalización. Se elaboró una lista organizada por área y descriptores temáticos, se identificaron 7 temas principales donde se agruparon los documentos, identificando posteriormente 28 temas específicos de forma homogénea encontrados en los documentos analizados.

En el indicador categorías metodológicas se evaluaron los enfoques y diseños de investigación, así como modelos de análisis que describen los firmantes en los documentos, se calculó a través de la misma óptica del análisis descripto en el párrafo anterior. 
Los datos obtenidos fueron analizados aplicando estadística descriptiva, utilizándose el cálculo de frecuencias y porcentajes. También se empleo la media y desviación estándar para los indicadores cuantitativos, el valor absoluto y porcentaje para los indicadores cualitativos. Se utilizó la medida de dispersión, clúster y medida de tendencia centra para determinar el punto de corte que establecen los diferentes grupos.

Para la visualización de los resultados más significativos se procedió a utilizar el software Microsoft Excel para la creación de tablas y figuras, asimismo se utilizó el programa UCINET 6.123 para generar las matrices y posteriormente obtener una visualización reticular mediante la utilización del programa NetDraw.

\section{Resultados y discusión}

Se presentan los resultados encontrados en esta investigación a través del análisis bibliométricos de los siguientes indicadores:

\subsection{Producción documental}

Desde el año 2006 hasta el año 2013 se publicaron en la revista ZT 349 artículos científicos, 20 notas técnicas y 11 resumen en extenso para un total de 380 documentos publicados, el número de artículos científicos y notas técnicas se han publicado de forma variable, los resúmenes en extenso en un solo año del período estudiado. Los artículos científicos van desde un máximo de 88 en 2008 hasta un mínimo de 24 en 2013 y las notas técnicas van desde un máximo 7 en 2013 y un mínimo de 1 entre 2008 y 2009. Podemos observa que en la mayoría de los años el número de artículos científicos está muy próximo a la misma y que la tendencia de la revista ZT reviste en la publicación de artículos científicos frete a otro tipo de trabajos (Figura 1), con una producción promedio de 35 documentos por año. Se percibe un ligero incremento de la productividad de documentos científicos que supera desde el 2006 las cifras correspondientes en ZT, un panorama distinto de lo que ocurría desde 1983 hasta 2005 en comparación con los estudios bibliométricos realizados por Pérez (Ob. Cit.), Arenas y Romero (Ob. Cit.) y, Chaparro y Marzal (Ob. Cit.) donde manifiestan que la producción promedio es de 30 documentos por año a partir de 1999. El hecho de que en el año 2007 se observe como el único periodo donde se publicaron resúmenes en extensos y que en el año 2008 se diera un aumento en el promedio de producción por año en los artículos científicos, obedece a la divulgación de los resultados presentados en las Primeras Jornadas Científico-Divulgativas y de Innovación realizadas por el Instituto Nacional de Investigaciones Agrícolas en el Estado Anzoátegui en el año 2007.

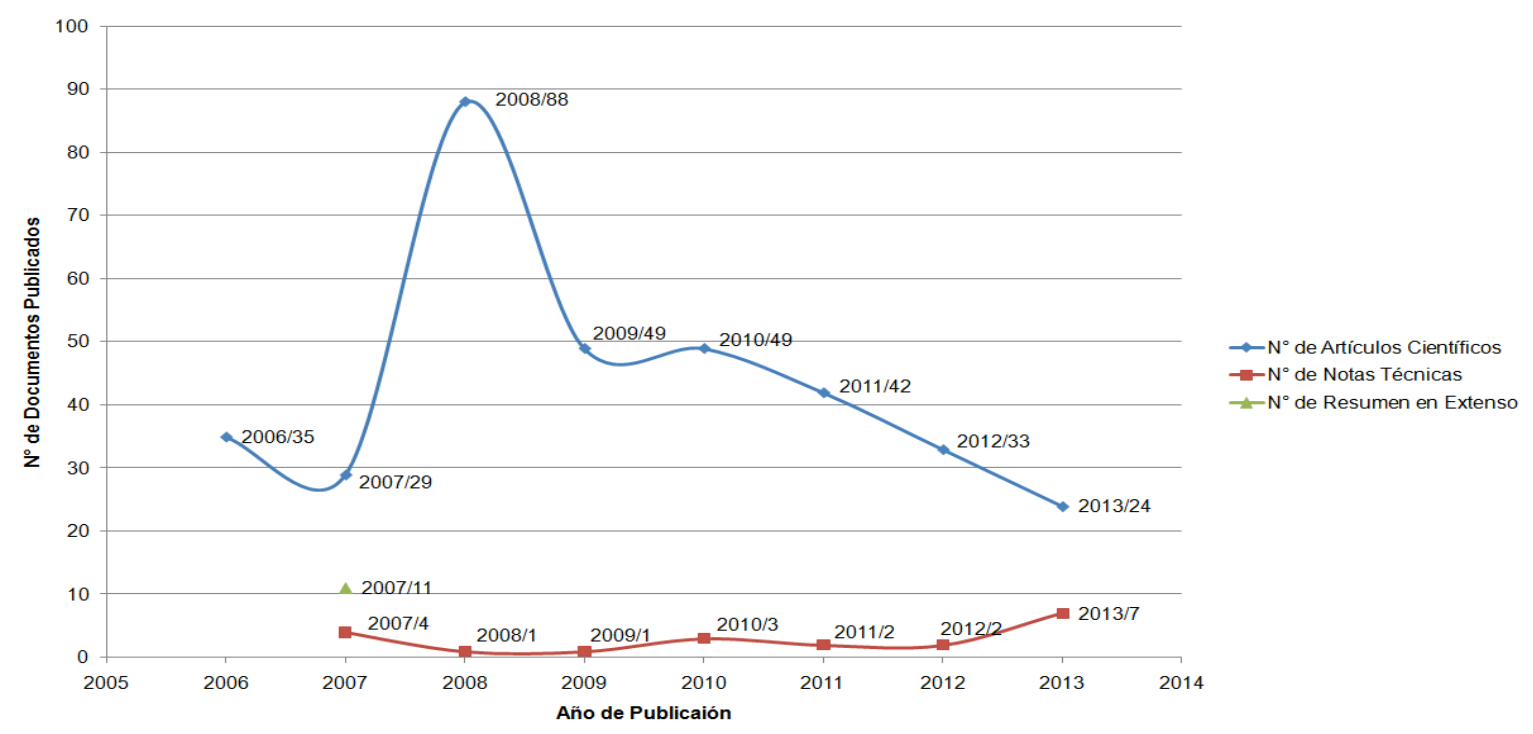

Figura 1- Producción de documentos publicados en la Revista ZT por año

\subsection{Productividad por países}


En la figura $\mathrm{N}^{\circ} 2$ se puede visualizar los resultados obtenidos en el indicador bibliométrico de publicación por procedencia geográfica de acuerdo al primer firmante, se identificó en la muestra un total de 10 países. Destacándose Venezuela con 243 publicaciones, seguidamente México con 46 publicaciones, Brasil con 25 publicaciones, Colombia con 17 publicaciones, Argentina con 10 publicaciones. En menor proporción Costa Rica con 4 publicaciones, Chile con 2 publicaciones y por último Nicaragua y Nigeria con 1 publicación cada uno, siendo los continentes americanos los que aportaron la mayor cantidad de publicaciones (99.74\%) y África Occidental una mínima cantidad $(0.26 \%)$. Un factor positivo de la productividad por países, tiene que ver con el incremento anual de las publicaciones de los países latinoamericanos y su impacto internacional en las sociedades discursivas de las ciencias agrícolas.

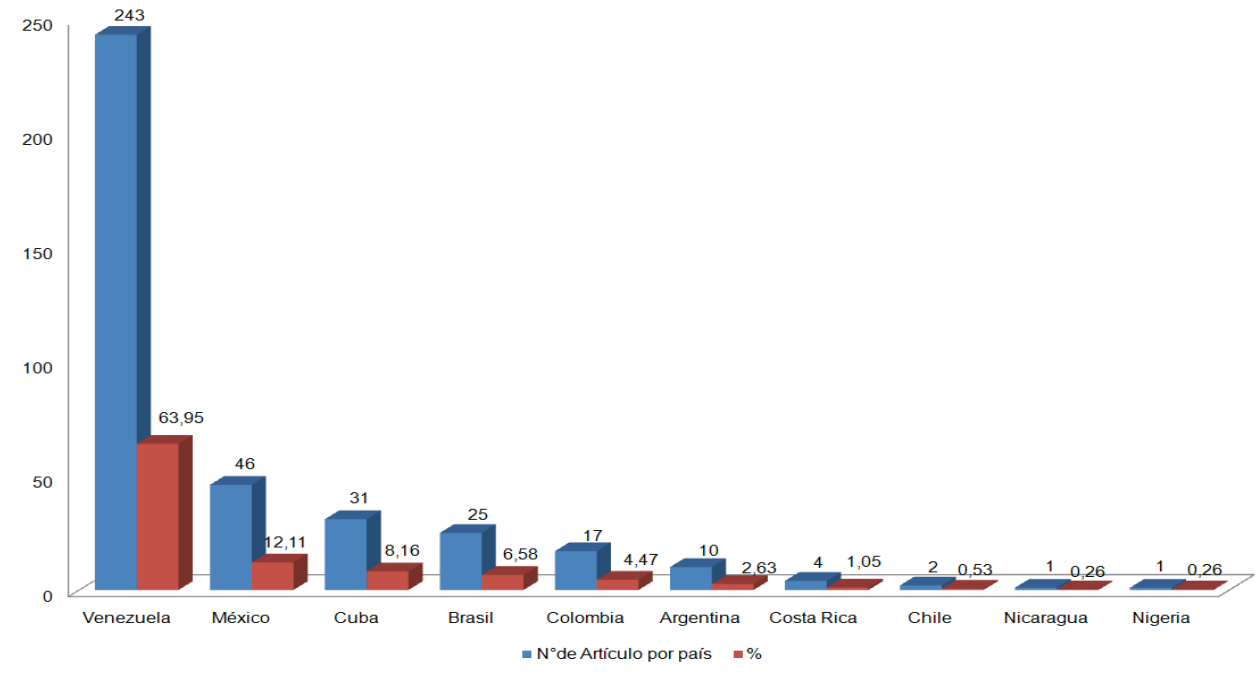

Figura 2 - Productividad por países en la Revista ZT

\subsection{Contribución por países}

Atendiendo al porcentaje de acuerdo a la colaboración del país de origen en los documentos publicados, se consideró el número total de nacionalidades de los autores firmantes, en su gran mayoría, suramericano y en una menor proporción europeo y asiático (Tabla 1), notándose en las publicaciones científicas un crédito para Venezuela, seguidamente Cuba, donde se pudo observar un crecimiento en el posicionamiento con respecto a lo reportado en las contribuciones por países en los tres estudios bibliométricos realizados anteriormente en ZT.

\begin{tabular}{lcc}
\hline País & $\mathrm{N}^{*}$ de Documentos & $\%$ \\
\hline Venezuela & 12 & 3,16 \\
Cuba & 11 & 2,89 \\
México & 8 & 2,11 \\
Colombia & 6 & 1,58 \\
España & 5 & 1,32 \\
Brasil & 4 & 1,05 \\
Argentina & 4 & 1,05 \\
Costa Rica & 2 & 0,53 \\
Chile & 2 & 0,53 \\
Camboya & 1 & 0,26 \\
Italia & 1 & 0,26 \\
Noruega & 1 & 0,26 \\
Alemania & 1 & 0,26 \\
Francia & 1 & 0,26 \\
\hline
\end{tabular}

Tabla 1 - Colaboración por países en la Revista ZT 


\subsection{Productividad institucional}

De acuerdo a la ley Lotka, Se identificaron 86 instituciones de carácter nacional e internacional conforme al primer firmante de los trabajos publicados, presentando una clasificación de tres niveles de rendimiento: índice de productividad transitoria (un único trabajo), donde encontramos 43 instituciones (50\%); índice de productividad intermedio (entre 2 y 9 trabajos) con 36 instituciones (41,86\%); e índice de productividad mayor (10 ó más trabajos) donde se localizaron 7 instituciones (8,14\%) que aportan el 59,17\% de los trabajos publicados (Tabla 2). Hay que matizar que la mayor contribución en la Revista ZT durante el periodo estudiado, es aportada por las universidades nacionales y extranjeras con un 70,94\% de las publicaciones y que los valores promedios reportados muestran que la institución líder de la investigación venezolana registrada en la Revista ZT es el INIA con una producción promedio del 25,52\%. La UCV es la segunda institución más significativa según su cuota de producción en el periodo 2006-2013 que representa el 10,52\% del total acreditado a Venezuela.

\begin{tabular}{|c|c|c|}
\hline Instituciones & $N^{\circ}$ de Documentos & $\%$ \\
\hline Instituto Nacional de Investigaciones Agrícolas & 97 & 25,52 \\
\hline Universidad Central de Venezuela & 40 & 10,52 \\
\hline Universidad Centroccidental "Lisandro Alvarado" & 27 & 7,10 \\
\hline Universidad de Oriente & 27 & 7,10 \\
\hline Universidad Nacional Experimental Rómulo Gallegos & 12 & 3,15 \\
\hline Estación Experimental de Pasto y Forrajes "Indio Hatuey" & 11 & 2,89 \\
\hline Universidad de los Andes & 11 & 2,89 \\
\hline Total $=$ & 225 & 59,17 \\
\hline
\end{tabular}

Tabla 2 - Productividad institucional en la Revista ZT

\subsection{Productividad científica y distribución de documentos publicados por el INIA}

La actividad científica del INIA por estado geográficos de Venezuela, registrada en los documentos publicados por la Revista ZT se observa en la Figura 3, de los 380 documentos analizados para esta investigación los firmantes del INIA participaron en 185 documentos con una participación multi-regional, siendo los firmantes adscripto a los estados Aragua, Trujillo, Lara, Yaracuy y Sucre los de mayor número de documentos publicados representándose en los nodos verdes; con una productividad intermedia se encuentra los firmantes pertenecientes a los estados Monagas, Zulia, Apure, Barinas, Falcón, Mérida, Delta Amacuro, Anzoátegui, Nueva Esparta, Guárico y Táchira representándose en los nodos amarillos; con una productividad transitoria se encuentra el estado Portuguesa representándose con el nodo rojo. En el análisis social de colaboración científica entre los firmantes adscriptos al INIA se observa en la red a través de los vínculos de color azul, notándose un solo nodo que no está conectado -Mérida-. Sin embargo, su propósito investigativo lo hace compartir con otras instituciones de carácter nacional. 


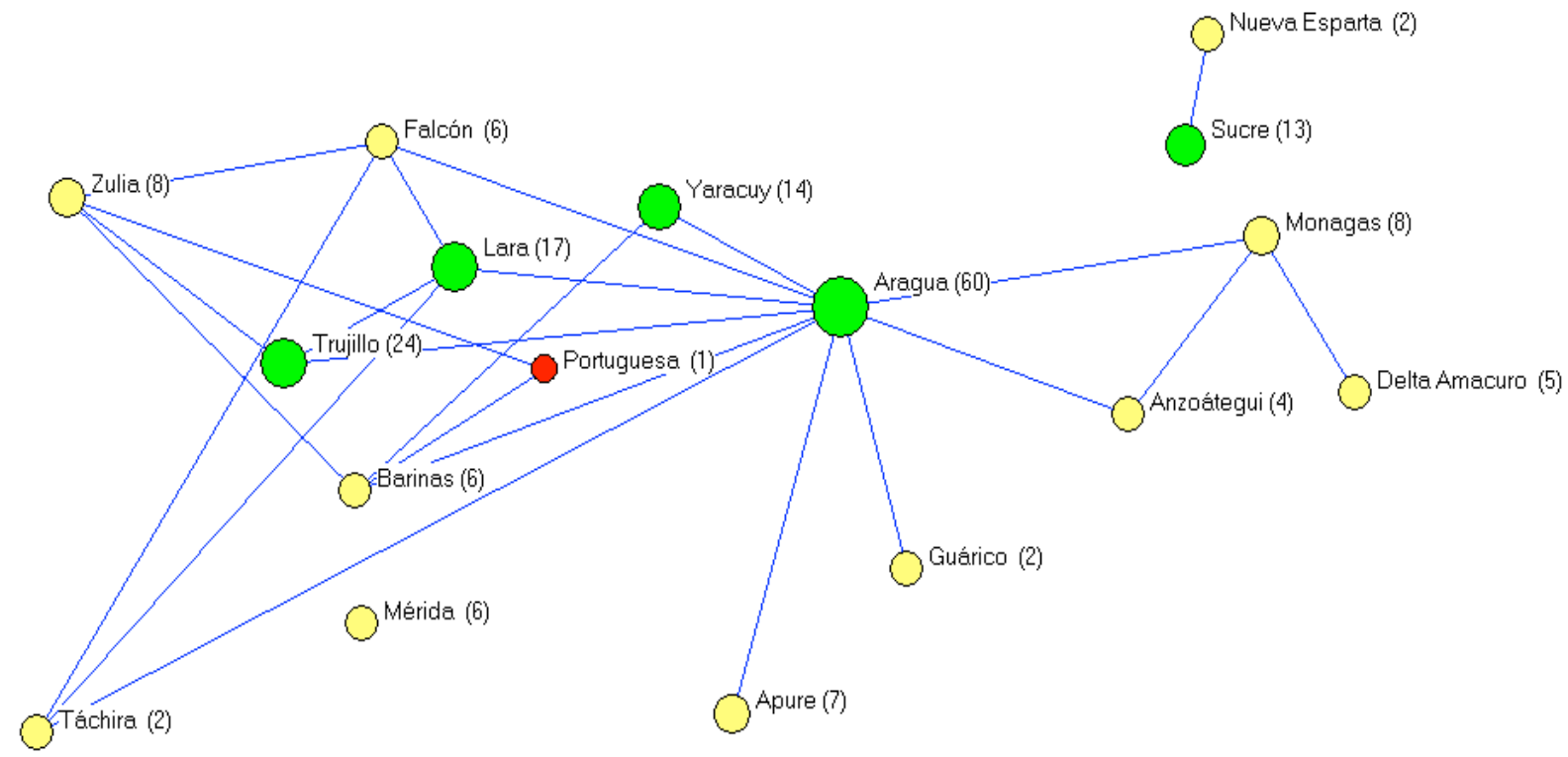

Figura 3 - Colaboración institucional y número de artículos por estados geográficos de Venezuela en Revista ZT

\subsection{Productividad autoral}

Luego de la normalización de los resultados se identificaron un total de 994 autores en la que se precisó que un gran número de investigadores tienen más de dos contribuciones realizadas en la revista ZT, sin embargo, a partir de la Ley de Lotka se distribuyeron los autores de acuerdo a los siguientes niveles de productividad:

\begin{tabular}{|l|c|c|c|}
\hline Nivel de productividad & \multirow{2}{*}{$\mathbf{N}^{\circ}$ de trabajos } & \multicolumn{2}{|c|}{ Autores } \\
\cline { 3 - 4 } & & $\mathrm{N}^{\circ}$ & $\%$ \\
\hline Grandes productores & 10 o más & 8 & $1 \%$ \\
\hline Medianos productores & 2 a 9 & 301 & $30 \%$ \\
\hline Pequeños productores & 1 & 685 & $69 \%$ \\
\hline \multicolumn{2}{|r}{ Total } & 994 & $100 \%$ \\
\hline
\end{tabular}

Tabla 3 - Distribución de autores de acuerdo al nivel de productividad

En la tabla 3 se puede observar que la gran mayoría de autores pertenecen al nivel de pequeños productores en la que se lograron identificar un total de 685 autores, lo que representa el 69\% de la totalidad de los productores, 3 de estos autores realizan su contribución de manera individual y 682 su participación es única como coautor. Por otro lado, los medianos productores representan el 30\% de los cuales 155 autores han realizado como mínimo 2 artículos y solo un autor ha realizo 9 contribuciones científicas, el resto se distribuye entre 5 a 8 artículos por autor. Solo una pequeña parte de 8 productores, se engloban dentro del nivel de grandes productores lo que representa el 1\% de la totalidad de los autores. En concordancia con los resultados presentados por Pérez (2003), Arenas (2003) y los resultados de la presente investigación los pequeños productores han mantenido este nivel de productividad entre el $60 \%, 61 \%$ y 69\% respectivamente, lo que sigue siendo de interés que este gran grupo de productores se conviertan en medianos productores, y como lo señala Arenas (2003) los mismos deben ser considerados autores potenciales de nuevos artículos y lograr llegar al nivel de medianos productores.

Por otro lado, en la tabla 4 se presentan los autores más productivos y que corresponde al nivel de productividad de grandes productores, así como también se ilustra la trayectoria de los cuatro primeros investigadores. 


\begin{tabular}{|c|c|}
\hline Autores & $\begin{array}{c}\text { Cantidad de } \\
\text { artículos }\end{array}$ \\
\hline Garcia, Danny & 26 \\
\hline Medina, María & 17 \\
\hline Lodeiros, César & 13 \\
\hline Morales, Gustavo & 13 \\
\hline Cova, Luís & 12 \\
\hline Principal, Judith & 12 \\
\hline Sandoval, Espartaco & 12 \\
\hline Acosta, Vanesa & 11 \\
\hline
\end{tabular}

Tabla 4 - Productividad autoral (Grandes Productores)

García, Danny. En función del período de estudio, el investigador contribuye a la revista a partir del año 2006 mostrando interés en los temas de los metabolitos secundarios en los bosques y en los materiales forrajeros, asimismo realiza sus investigaciones en años posteriores enfatizando en la importancia de la morera como alimentación alternativa para la alimentación animal sustentando sus estudios en aspectos conceptuales, metodológicos y prácticos. Sus últimas investigaciones en colaboración se enmarcan en el uso de implantes anabólicos como el Zeranol como estrategia para la engorda intensiva de bovinos y en el ranking del presente estudio ocupa el primer lugar con 24 contribuciones científicas. García actualmente es asesor de la revista de la Facultad de Agronomía de la Universidad del Zulia en el área de la Forrajicultura y se desempeña como investigador del INIA en el estado Trujillo.

Medina, María. Con un total de 15 contribuciones científicas, la Ingeniera Medina es instructor a tiempo completo en el área de Agroecología y Extensión adscrito al Departamento de Ciencias Agrarias de la Universidad de Los Andes. Entre las investigaciones más significativas se encuentran dos artículos publicados en coautoría en el año 2006 y uno en el 2009, donde las mismas son citadas al año siguiente, que en términos bibliométricos cumple con los parámetros del índice de inmediatez, cuyas publicaciones son citadas al año siguiente de su publicación; entre los artículos que tienen mayor citas se encuentran el artículo "Composición química, metabolitos secundarios, valor nutritivo y aceptabilidad relativa de diez árboles forrajeros" y el artículo "Variables morfo-estructurales y de calidad de la biomasa de Tithonia diversifolia en la etapa inicial de crecimiento" con un total de 43 y 21 citas respectivamente.

Lodeiros, César. El investigador Lodeiros posee una gran trayectoria en la docencia e investigaciones en el área de la acuicultura y hoy en día posee un centenar de publicaciones en revistas científicas de gran impacto, así como libros y capítulos de libros en el área de Ciencias Marinas, resaltando sus estudios en la acuicultura, ecología y fisiología en moluscos bivalvos. Para efecto de esta investigación el Doctor Lodeiros contribuyó con 13 producciones científicas ocupando un puesto previligiado entre los grandes productores. Es importante destacar que el investigador en el año 2011 recibe el Premio Lorenzo Mendoza Fleury, uno de los más importantes galardones que se conceden en el área en Venezuela, asimismo en el año 2013 recibe el Premio Interciencia, galardon auspiciado por la Asociación Venezolana para el Avance de la Ciencia (AsoVAC). Desde 1988 el Profesor César Lodeiros Seijo realiza investigaciones en el área de las Ciencias Marinas como personal adjunto al Dpto. de Biología Pesquera del Instituto Oceanográfico de Venezuela de la Universidad de Oriente (IOV-UDO). Desde 1989 es profesor del postgrado en Ciencias Marinas (M.Sc. y Ph.D.) que el IOV-UDO imparte.

Morales, Gustavo. El investigador Morales es médico veterinario, con postgrado en Patología Animal Tropical (Francia) y Doctor en Ciencias (Universidad de París VI); fue profesor en el Núcleo Universitario Rafael Rangel, y actualmente trabaja como investigador en el INIA, estado Aragua. Morales es un estudioso de la parte parasitológica y con él se iniciaron en Trujillo las investigaciones de Distomatosis Bovina, es decir la Fasciolasis Hepática. Su última investigación, en la revista ZT, la realizó abordando un estudio sobre la estimación del riesgo de la presencia de anemia en becerros nacidos de vacas anémicas durante el periparto. 


\subsection{Colaboración autoral}

En la figura 4 se presentan las relaciones de colaboración entre las firmas identificadas a partir de los 380 artículos recuperados. Se seleccionaron aquellos artículos firmados por más de un autor y se obtuvo las relaciones más significativas que son las que se muestran a continuación:

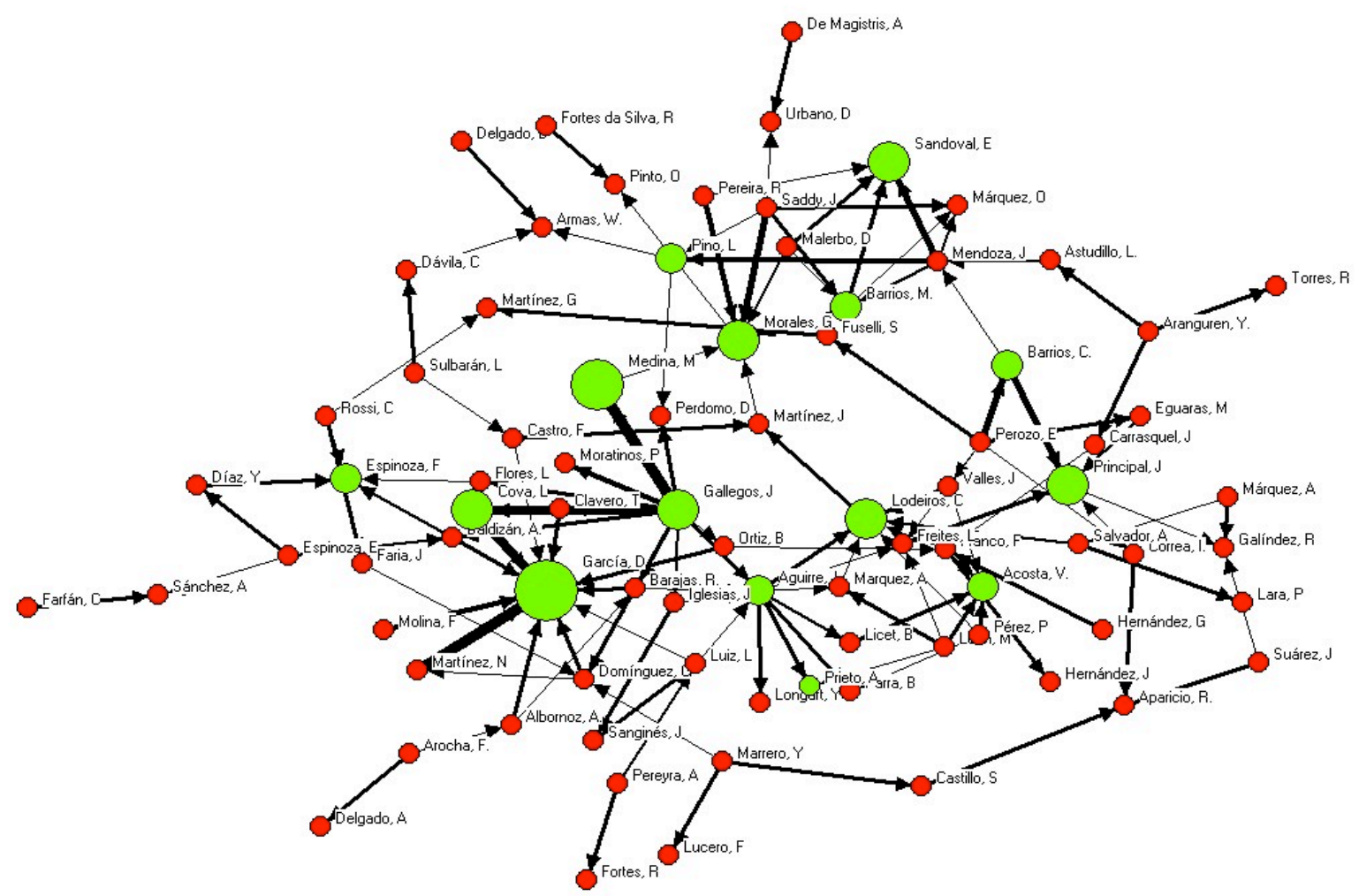

Figura 4 - Colaboración autoral Revista ZT

En el análisis de este indicador se puede apreciar que es una red conectada, en la que se puede distinguir diversos grupos estrechamente relacionados que comparten intereses comunes de conocimiento. Existe una alta colaboración entre los autores más productivos (nodos verdes), así como también una correspondencia entre los autores más productivos con lo que más colaboran, lo cual valida el interés de estos investigadores por la diversidad temática en el área de la agropecuaria. Entre los autores que más colaboran están García, Martínez, Cova, Gallegos y Medina en la que se puede observar claramente a partir de las fuertes relaciones que se aprecian en los enlaces. Se puede observar además, que los autores de menor colaboración generan otro grupo cuyas relaciones no son tan débiles. Respecto a los autores venezolanos, estos prefieren publicar entre tres o más autores.

\subsection{Colaboración en las publicaciones}

A continuación se muestra la distribución del índice de firmas/documentos publicados de acuerdo al análisis estadístico longitudinal del periodo estudiado. El índice de firmas se mantiene constante entorno a los documentos publicados por año, encontrándose en 4,11 y 4,90 autores por documento y la media de todo el periodo es 4,43 autores por documento, (Tabla 5). Las publicaciones con un solo autor disminuyen y ocupan una mínima posición. Desde el año 2006 hasta 2013 se registraron artículos firmados por 2 o más autores. El mayor número de firmantes para un artículo es de 11, registrado en el año 2008, Volumen 6, número 3. Se considera un signo tangible y está acorde con la tendencia internacional que incrementa el factor de publicar en equipos de investigación, donde cada autor debe participar en el trabajo para poder asumir su responsabilidad por el contenido del artículo (Sancho,Ob. Cit.). 


\begin{tabular}{|l|c|c|c|c|c|c|c|c|c|}
\hline Año de publicación & 2006 & 2007 & 2008 & 2009 & 2010 & 2011 & 2012 & 2013 & Total \\
\hline $\mathrm{N}^{\circ}$ de Firmas & 152 & 200 & 403 & 213 & 255 & 181 & 155 & 133 & 1.692 \\
\hline $\mathrm{N}^{\circ}$ de Documentos & 35 & 44 & 89 & 50 & 52 & 44 & 35 & 31 & 380 \\
\hline Índice Anual de Firma/Documento & 4,34 & 4,55 & 4,53 & 4,26 & 4,90 & 4,11 & 4,43 & 4,29 & 4,43 \\
\hline
\end{tabular}

Tabla 5 - Número de firmas/documentos

\subsection{Capacidad idiomática}

En el análisis de la producción idiomática en la Revista ZT predomina el idioma español con una constante de 352 publicaciones, le sigue el idioma portugués con 21 publicaciones y por último el idioma inglés con 7 publicaciones (Figura 5). Sin embargo es necesario destacar que los documentos publicados en idioma inglés no son producidos por nativos de habla inglesa, son generados por autores con mayor capacidad idiomática y tienden a publicar artículo en el idioma inglés. Estos valores evidencian la preferencia de los firmantes por el idioma español para sus publicaciones, como vehículo de la comunicación científica.

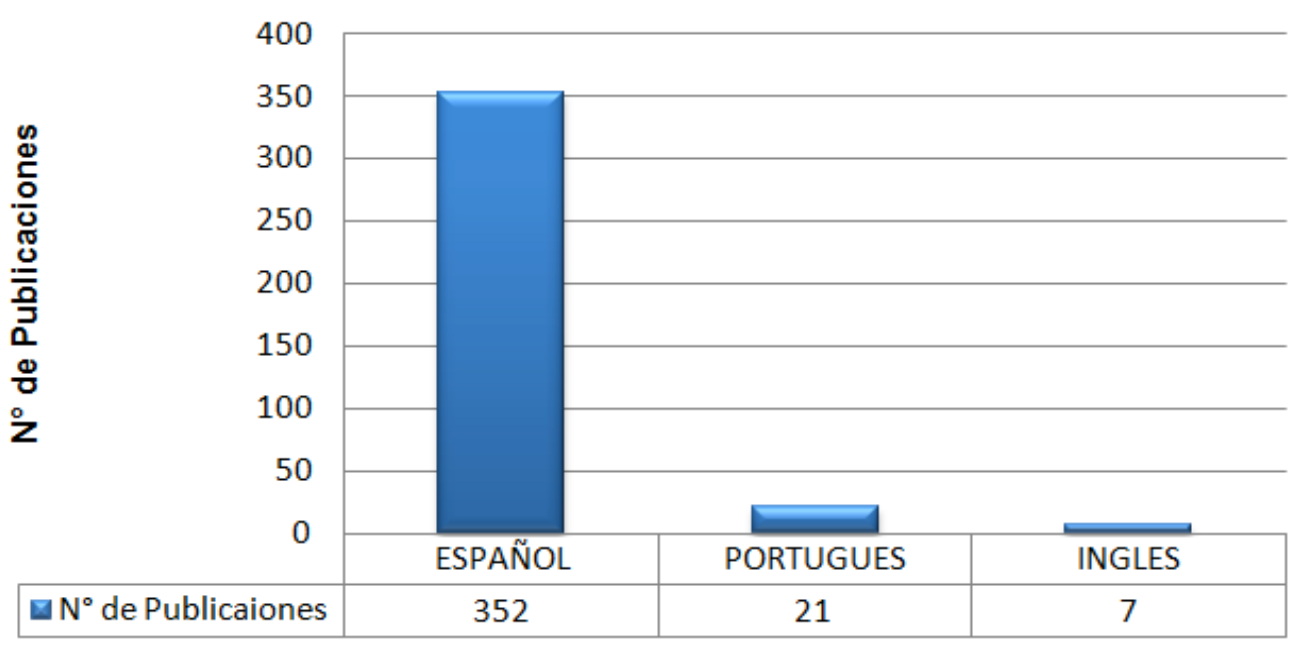

Figura 5 - Capacidad idiomática de los documentos publicados en la Revista ZT

\subsection{Categorías temáticas}

La identificación de los temas de investigación desarrollada en la Revista ZT se puede visualizar en el índice de cohesión externa en la figura 6. Se aprecia en núcleo del grafo -temáticas en ZT- los vínculos con 7 temas principales de investigación representados por los nodos verdes, nótese que todos son del mismo grado nodal -tamaño-, permitiendo la incorporación de los temas precisados en la investigación y representados en la red por los nodos rojos. Se concretaron en la red 35 subgrupos (cliques) para delimitar con mayor exactitud la productividad de investigación temática que se establece de acuerdo al análisis del dominio, el análisis de co-palabras, el Sistema AGRIS, el código de objeto AGRIS y el tesauro AGROVOC. Las temáticas de investigación se pueden apreciar en los nodos de color rojo y su dominio se puede distinguir por el mayor grado nodal. Obsérvese que en los temas motores del campo científico alimentación animal y ganadería es mayor la centralidad y densidad en la red. Esto significa que son los temas que mayor coherencia interna se presentan en este período analizado. Resulta significativo que el tema fisiología y bioquímica de la planta aunque aparezca en una posición de periferia en la red y que sus valores de centralidad y densidad es bajo, mostró que es el que mayor número de artículo acumula en este periodo estudiado con respecto a los temas centrales e inherente al campo científico estudiado, evidenciando un interés en los investigadores en perfeccionar métodos de evaluación en las plantas para analizar su valor nutricional. Es así como los estudios se han enfocado en adaptar y validar instrumentos que sirvan a la ciencia y producción vegetal como soporte de un adecuado análisis, siendo este un tema coherente y perteneciente a un área específica. Otra área temática de importancia que se muestra en este estudio es la pesca y acuicultura que son temas centrales y pocos desarrollados, estos resultados coinciden con el estudio bibliométrico realizado por Arenas y Romero (Ob. Cit.). En la sub categoría de materia se debe tener en cuenta que existen unas áreas que aún faltan por investigar a profundidad 
como son la ecología acuática, producción pesquera y composición de los alimentos, pues, como se puede observar en el estudio, son las áreas más rezagadas. Los resultados obtenidos evidencian que los documentos publicados sobre la ciencia, producción y protección animal mostraron valores más bajos con respecto a los temas específicos publicados, lo que dificulta la generación de investigaciones que funden líneas de trabajo a desarrollar para la producción animal. Predominan artículos de corte práctico a partir de las experiencias surgidas de la introducción de técnicas relacionadas con la ciencia y producción vegetal.

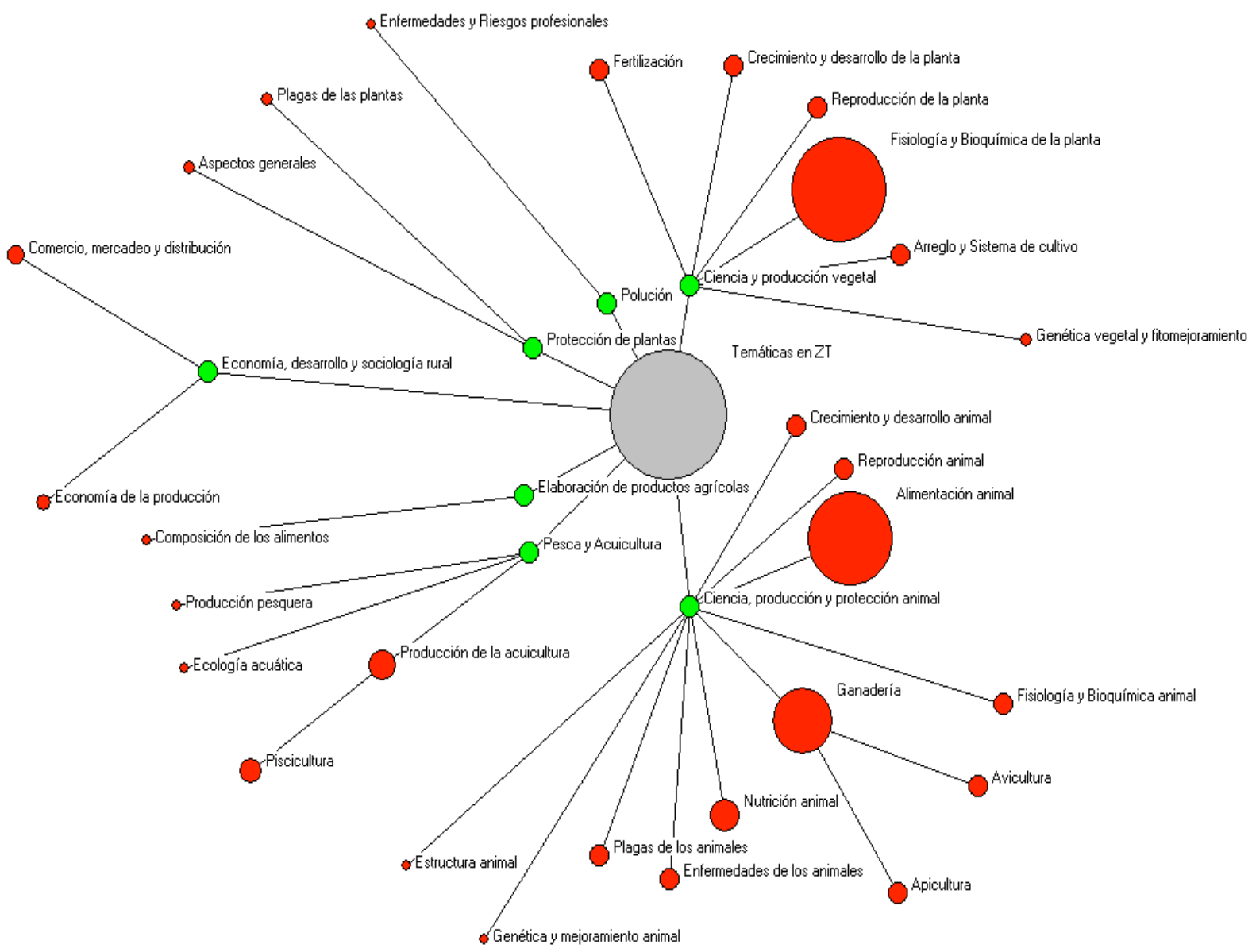

Figura 6 - Temas de investigación desarrollado en la Revista ZT

\subsection{Categorías metodológicas}

En relación a la metodología de investigación es conveniente señalar que los temas analizados muestran que el enfoque cuantitativo es el dominante (380) y que dentro de la variedad de diseños de investigativos, el diseño de investigación descriptivo (318) produjo el mayor número de trabajos, seguido del diseño de investigación mixto (34) e inferencial (28). Adicionalmente, se encontró dentro de los documentos publicados que el modelo de análisis estadístico más relevante es el multivariado (41,05\%) y con un menor porcentaje los análisis de: varianza (18,16\%), frecuencia (7,89\%). Dentro del diseño de investigación mixto el método de análisis dominante es multivariado (8.95\%) y en el diseño de investigación inferencial el método de análisis dominante es Chi-cuadro $(3,16)$. Esto demuestra que la tendencia se centra en realizar trabajos empíricos que trabajos teóricos. Toda esta matematización se puede ver por el grado nodal -tamaño- y por la densidad de la relaciones entre los nodos en la figura 7. No obstante, es impórtate señalar que también existen fortalezas para desarrollar trabajos de investigación desde el enfoque cualitativo que, aunque no se refleja en los resultados de este trabajo, igualmente aparece como el otro enfoque metodológico para ser utilizado de acuerdo a las instrucciones para los autores de la Revista ZT. Esto pone de relieve al INIA para analizar toda producción científica del área agrícola. 


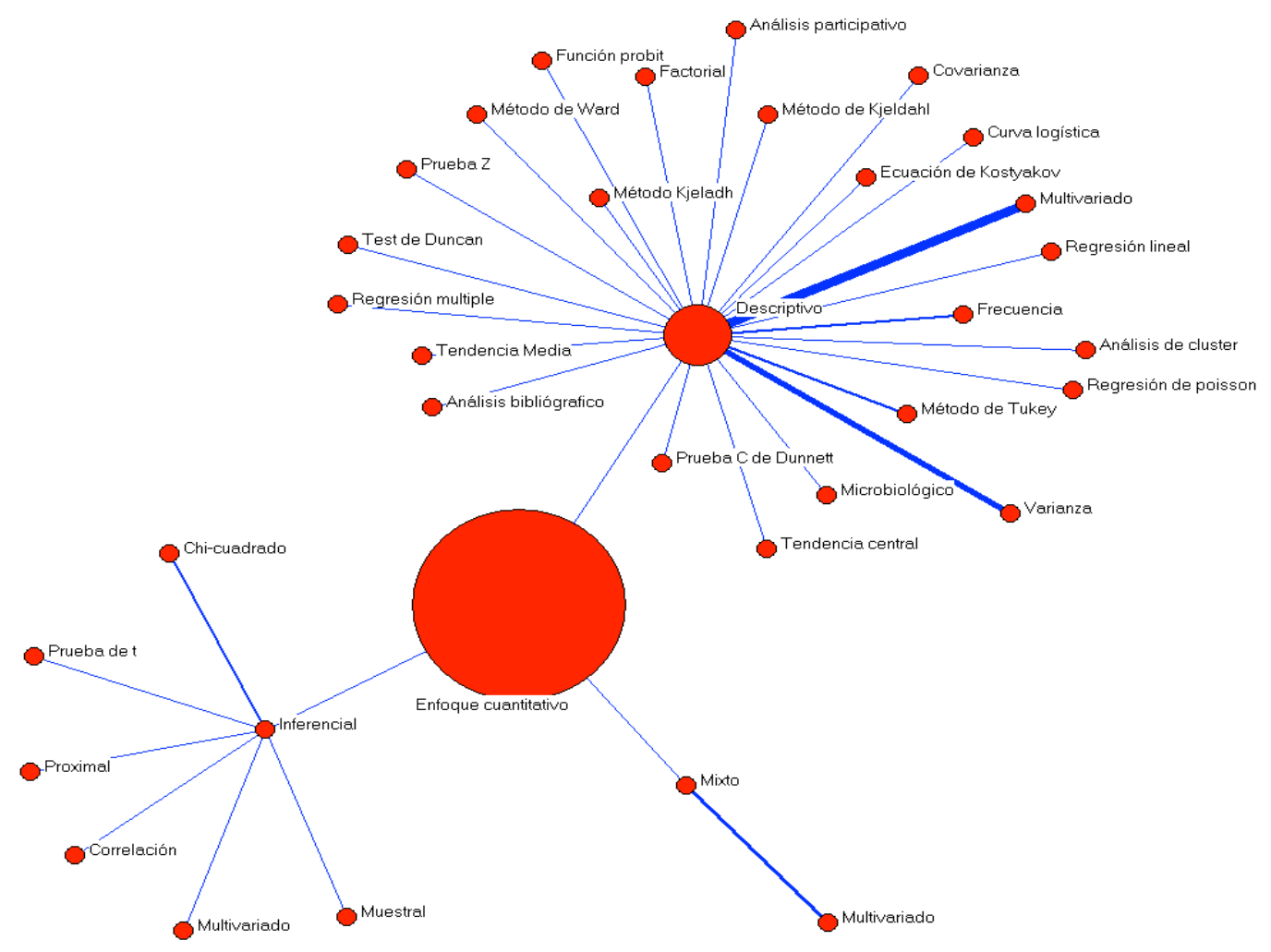

Figura 7 - Metodología de la investigación registrada en los documentos de la Revista ZT

\section{Conclusiones}

De acuerdo al análisis bibliométrico de los artículos de la Revista Zootecnia Tropical del Instituto Nacional de Investigaciones Agrícolas durante el periodo 2006-2013, existe un incremento en la publicación de documentos científicos en comparación con los estudios bibliométricos realizados anteriormente a este trabajo.

En la investigación se comprobó que en conjunto, El Instituto Nacional de Investigaciones Agrícolas, La Universidad Central de Venezuela, La Universidad Centroccidental "Lisandro Alvarado", La Universidad de Oriente, La Universidad Experimental Rómulo Gallegos y la Universidad de los Andes contribuyeron con más de 50 \% en la producción científica en el área y en un menor porcentaje las instituciones internacionales.

Se aprecia una comunidad científica conectada y relacionada de acuerdo a los intereses comunes del conocimiento, prevaleciendo el idioma español como vehículo de comunicación científico en la Revista ZT.

Sobre las temáticas registradas en las publicaciones de la Revista ZT durante el periodo estudiado, se pudo observar que existe una mayor centralidad y densidad en 2 de los temas motores del campo científico. Predominaron los temas de Fisiología y Bioquímica de la Planta, siendo sus valores de centralidad y densidad bajos. No obstante, estos elementos pueden aumentar con el seguimiento del comité editor y de los pares revisores de los artículos que se someten a consideración de la revista.

Se evidencia que el enfoque cuantitativo tiene un posicionamiento hegemónico en la productividad científica de la Revista ZT. Sin embargo, se puede establecer como una oportunidad el producir investigaciones desde un enfoque cualitativo.

La aplicación del análisis del dominio y análisis de co-palabras como métodos constituyen herramientas importantes para evaluar la producción científica que propicia evidencias importantes para trazar políticas de publicación y contribuir al fortalecimiento de la comunidad científica en torno a los temas de investigación priorizados y necesarios para el desarrollo agrícola del país. 


\section{Referencias}

Arenas, S. y Romero, A. (2003). Indicadores bibliométricos de la revista científica Zootecnia Tropical. Rev. Zootecnia Tropical. 21(3).

Callon, M., Law, J., \& Rip, A. (1986). Mapping the Dynamics of Science and Technology: Sociology of Science in a Rreal World. The Macmillan Press Ltd. London.

Chaparro, M, E. y Marzal M. Á. (2009). Analysis of bibliographic references in Zootecnia Tropical ", Library Review, 58 (6).

Chen, C. (2003). Mapping Scientific Frontiers: The Quest for K knowledge Visualization. Springer. London.

FAO. (1997). AGROVOC. Tesauro Agrícola Multilingüe. 3ra Ed. Organización de las Naciones Unidas para la Alimentación y la Agricultura. Roma, Italia.

FAO-CARIS-7. (1990). AGRIS/CARIS: Esquema de categoría de Materias. FAO-AGRIS-3. (Rev.5). Es. Dirección de la biblioteca y sistemas de documentación. Organización de la Naciones Unidas para la Agricultura y la Alimentación. Roma, Italia.

Hjørland, B. y Albrechtsen, H. (1995). Toward a new horizon in Information and Science Domain Analysis. Journal of the American Society for Information Science, 46(6). Recuperado de http://www.mendeley.com/research/toward-new-horizon-informationscience-domain-analysis-1/

Lotka, A.J. (1926). The frequency distribution of scientific productivity. Journal of the Washington Academy of Sciences, 16 (12).

Montilla, L. (2012). Análisis bibliométrico sobre la producción científica archivística en la Red de Revistas Científicas de América Latina y el Caribe (Redalyc) durante el período 2001-2011. Biblios. 48. recuperado de http://biblios.pitt.edu/ojs/index.php/biblios/article/view/65/128

Pérez, G. (2014). Análisis bibliométrico a la Revista Enlace Científico, período 1999-2010. Enlace Científico. 15 (10).

Pérez, S. M. (2003). Análisis temático y consumo de información de la revista Zootecnia Tropical para el período 1983-2002. Trabajo de grado Ingeniero Agrónomo. Facultad de Agronomía. Universidad Central de Venezuela. Maracay.

Rubio, M. 1999. Bibliometría y ciencias sociales. Proyecto Clio. 7. Disponible en http://clio.rediris.es/articulos/bibliometria.htm

Sancho, R. (1990). Indicadores bibliométricos utilizados en la evaluación de la ciencia y la tecnología. Revisión bibliográfica. Rev. Esp. Doc. Cient. 13 (3-4).

Spinak, E. (1996). Diccionario Enciclopédico de Bibliometría, Cienciometría e Informetría. UNESCO. Caracas. .

Torres, D. (2010). La visualización de la información en el entorno de la Ciencia de la Información. Tesis Doctoral para el grado de Doctor en Documentación e Información Científica. Faculta de Comunicación y Documentación. Universidad de Granada. 


\section{Datos del autor}

\section{Leomar José Montilla Peña}

Máster en Bibliotecología y Ciencias de la Información. Egresado de la Universidad de la Habana, Faculta de Comunicación, La Habana, Cuba. Licenciado en Bibliotecología y Ciencias de la Información por la misma universidad. Encargado de la Biblioteca Especializada del Instituto Nacional de Investigaciones Agrícola, Unidad Ejecutora Lara, Venezuela. Profesor del Programa Nacional de Formación en Ciencias de la Información en la Universidad Politécnica Territorial de Lara Andrés Eloy Blanco, Barquisimeto, Venezuela. Integrante del Comité Interinstitucional para el Fortalecimiento de la Gestión del Programa Nacional de Formación en Ciencias de la Información, Ministerio del Poder Popular para la Educación Universitaria, Caracas, Venezuela.

leomonp@gmail.com

\section{Guillermo Andrés Pérez Reyes}

Máster en Bibliotecología y Ciencias de la Información. Egresado de la Universidad de la Habana, Facultad de Comunicación, La Habana, Cuba. Licenciado en Bibliotecología y Ciencias de la Información por la misma universidad. Jefe de la Biblioteca de la Universidad Politécnica Territorial Andrés Eloy Blanco Barquisimeto, Venezuela. Profesor Instructor del Programa de Formación en Ciencias de la Información de la Universidad Politécnica Territorial Andrés Eloy Blanco, Barquisimeto, Venezuela. Integrante del Comité Interinstitucional para el Fortalecimiento de la Gestión del Programa Nacional de Formación en Ciencias de la Información, Ministerio del Poder Popular para la Educación Universitaria, Caracas, Venezuela.

pguillermoandres@gmail.com

Recibido - Received: 2016-04-14

Aceitado - Accepted: 2016-07-12

\section{(cc) BY}

This work is licensed under a Creative Commons Attribution 4.0 United States License.

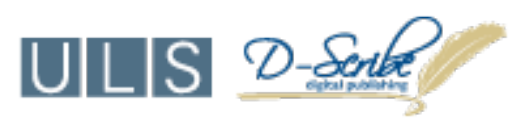

This journal is published by the University Library System of the University of Pittsburgh as part of its D-Scribe Digital Publishing Program and is cosponsored by the University of Pittsburgh Press. 